\title{
Minima Domain Intervals and the $S$-Convexity, as well as the Convexity, Phenomenon
}

\author{
I. M. R. Pinheiro \\ P.O. Box 12396 A'Beckett St, Melbourne, VIC, AU, 8006 \\ Email: illmrpinheiro@gmail.com
}

Received July 29, 2011; revised August 29, 2011; accepted September 12, 2011

\begin{abstract}
In this paper, we propose a refinement in the analytical definition of the $s_{2}$-convex classes of functions aiming to progress further in the direction of including $s_{2}$-convexity properly in the body of Real Analysis.
\end{abstract}

Keywords: Analysis; Convexity; Definition; S-Convexity

\section{Introduction}

We have been working on refining and improving, as well as fixing, the definition of the $s_{2}$-convex classes of functions for a while now (since 2001). The concept $S$ convexity is supposed to be a proper extension of the concept Convexity, what then implies that some of our proposed modifications may, if "universally" accepted, affect the definition of the convex class of functions.

This piece of work presents argumentation in favor of an explicit reference to a minimum necessary interval of domain in the analytical definition of the $s_{2}$-convex classes of functions.

The sequence of presentation of this paper is:

- Introduction;

- Analytical definition of the phenomenon $s_{2}$-convexity and symbology;

- Geometric definition of the phenomenon $s_{2}$-convexity;

- Argumentation defending an explicit reference to a minimum interval of domain in the analytical definition of the $s_{2}$-convex class of functions;

- Proposed definition;

- Conclusion;

- References.

\section{Analytical Definition of the Phenomenon $s_{2}$-Convexity and Symbology}

\subsection{Symbols [1]}

- $K_{s}^{2}$ stands for the set of $S$-convex classes of type 2;

- $0<s \leq 1$ is the real number to replace $s_{2}$ in the expression " $s_{2}$-convex", therefore the number to actually select one class amongst the so many possible $s_{2}$-convex classes of functions;
- $K_{1}^{2}$ is a mathematical synonym for the class convex functions.

\subsection{Definitions [2]}

Definition 1. A function $f: X_{-}>\mathfrak{R}$, where $|f(x)|=$ $f(x)$, is told to belong to $K_{s}^{2}$ if the inequality

$$
f(\lambda x+(1-\lambda) y) \leq \lambda^{s} f(x)+(1-\lambda)^{s} f(y)
$$

holds $\forall \lambda / \lambda \in[0,1] ; \forall\{x, y\} /\{x, y\} \subset X$; $s=s_{2} / 0<s_{2} \leq 1 ; \quad X \subseteq \mathfrak{R}_{+}$.

Definition 2. A function $f: X_{-}>\mathfrak{R}$, where $|f(x)|=$ $-f(x)$, is told to belong to $K_{s}^{2}$ if the inequality

$$
f(\lambda x+(1-\lambda) y) \leq \lambda^{\frac{1}{s}} f(x)+(1-\lambda)^{\frac{1}{s}} f(y)
$$

holds $\forall \lambda / \lambda \in[0,1] ; \forall\{x, y\} /\{x, y\} \subset X$; $s=s_{2} / 0<s_{2} \leq 1 ; \quad X \subseteq \mathfrak{R}_{+}$.

Remark 1. If $f^{1}$ the inequality is obeyed in the reverse ${ }^{2}$ situation by $f$, then $f$ is said to be $s_{2}$-concave.

\section{Geometric Definition of the Phenomenon $s_{2}$-Convexity}

Definition 3. A real function $f: X_{-}>Y$ is called convex if and only if, for all choices $\left(x_{1} ; y_{1}\right)$ and $\left(x_{2} ; y_{2}\right)$, where $\left\{x_{1}, x_{2}\right\} \subset X,\left\{y_{1}, y_{2}\right\} \subset Y, Y=\operatorname{Im}_{f}$, and $x_{1} \neq$ $x_{2}$, it happens that the chord drawn between $\left(x_{1} ; y_{1}\right)$ and $\left(x_{2} ; y_{2}\right)$ does not contain any point with height, measured against the vertical cartesian axis, that be inferior to the height of its horizontal equivalent in the curve representing the ordered pairs of $f$ in the interval considered for the chord.

\footnotetext{
${ }^{1}$ This remark applies to both definitions preceding it.

${ }^{2}$ Reverse here means $>$, not $\geq$.
} 
Remark 2. Notice that, to extend the above geometric definition, one needs to come up with a limiting geometric line connecting the same set of points $\left(\left(x_{1} ; y_{1}\right)\right.$, $\left.\left(x_{2} ; y_{2}\right)\right)$, but a line that lie above the chords in at least some piece of it. Furthermore, it is clearly the case that one must have a continuous line as curve for $f$ (otherwise, we give margin to doubts in the mathematical decisions to be made when comparing the chord with the line), what then implies that if a real function $f$ is $s_{2}$-convex in $[a, b]$, then it is continuous in $[a, b]$.

\section{Argumentation Defending an Explicit Reference to a Minimum Interval of Domain in the Analytical Definition of the $s_{2}$-Convex Class of Functions}

One of the major criticisms to $S$-convexity is that clear inconsistency is found when putting the geometrical definition against the analytical one.

The geometric definition of Convexity, therefore also of S-convexity, DOES imply that the right side of the inequality forms a line, not mattering if curved or straight. The only way to generate this line, minimum condition of existence for it, is that $x \neq y$, therefore $X \neq\{x\}$, $\forall x \in \Re$.

One needs more than one point to form a line; in fact, an infinite number of points is needed, therefore an interval, a minimum interval. Because we do not find mention to such an interval so far in the definitions, there is a clear omission in the analytical definition of S-convexity, therefore in the definition of convexity.

The necessity of the minimum interval is quite obvious: $x$ must be different from $y$ in all definitions (coherence of the geometric and analytical definitions) and the mention to a piece $X$, from the real numbers, usually excludes the possibility of $X$ being a degenerated interval (elegance).

Nothing could be more appropriate than adding a "distance element" to the current analytical definition, therefore.

Let's call this distance element $\delta$.

Given the nature of $\delta$, read from the previous paragraphs, it can only be that $\delta>0$.

Notice that the current definition of S-convexity deals with a vector in $\mathfrak{R}^{2}((x, y))$, but its current domain is in $\Re$ and so we want it to be in order to have the analytical definition matching the geometrical one.

To take away one dimension from the definition, it suffices that we replace $y$ with $(x+\delta)$, action that will also address our minimum interval problem.

\section{Proposed Definition}

Definition 4. A function $f: X_{-}>\Re$, where $|f(x)|=$ $f(x)$, is told to belong to $K_{s}^{2}$ if the inequality

$$
f(\lambda x+(1-\lambda)(x+\delta)) \leq \lambda^{s} f(x)+(1-\lambda)^{s} f(x+\delta)
$$

holds $\forall \lambda / \lambda \in[0,1] ; \quad \forall x / x \in X ; s=s_{2} / 0<s_{2} \leq 1$; $X / X \subseteq \Re_{+} \wedge X=[a, b] ; \forall \delta / 0<\delta \leq(b-x)$.

Definition 5. A function $f: X_{-}>\Re$, where $|f(x)|=$ $-f(x)$, is told to belong to $K_{s}^{2}$ if the inequality

$$
f(\lambda x+(1-\lambda)(x+\delta)) \leq \lambda^{\frac{1}{s}} f(x)+(1-\lambda)^{\frac{1}{s}} f(x+\delta)
$$

holds $\forall \lambda / \lambda \in[0,1] ; \quad \forall x / x \in X ; s=s_{2} / 0<s_{2} \leq 1$; $X / X \subseteq \Re_{+} \wedge X=[a, b] ; \forall \delta / 0<\delta \leq(b-x)$.

Remark 3. If the inequality is obeyed in the reverse $e^{4}$ situation by $f$, then $f$ is said to be $s_{2}$-concave.

\section{Conclusions}

In this short note, we have proposed more modifications to the analytical definition of the phenomenon S-convexity.

This time, our proposed modifications, if accepted, will affect the number of variables and the domain interval in the definition of $S_{2}$-convexity.

Our proposed wording for the definition of the phenomenon $S_{2}$-convexity brings $(x+\delta)$ in place of $y$ and limits $\delta$ in order to guarantee both that the domain interval be not degenerated and that $(x+\delta)$ be inside of the boundaries of the domain.

\section{REFERENCES}

[1] M. R. Pinheiro, "Convexity Secrets," Trafford Publishing, England, 2008. ISBN: 1425138217.

[2] M. R. Pinheiro, "First Note on the Definition of $s_{2}$-Convexity," Advances in Pure Mathematics, Vol. 1, No. 1, 2011, pp. 1-2. 